ARTICULO ORIGINAL

\title{
Estudio bibliométrico de la producción científica de los docentes investigadores del Instituto de Investigaciones en Ciencias de la Salud. Periodo 1997 - 2011
}

\section{Bibliometric study of the scientific output of the researchers of the Instituto de Investigaciones en Ciencias de la Salud. Period 1997 - 2011}

\author{
*Torales Cabañas $\mathrm{M}^{\mathrm{I}}$, Leguizamón MA ${ }^{\mathrm{II}}$, Samudio $\mathrm{M}^{\mathrm{III}}$ \\ ${ }^{\text {I}}$ Perceptoria. Instituto de Investigaciones en Ciencias de la Salud. Universidad Nacional de \\ Asunción, Paraguay

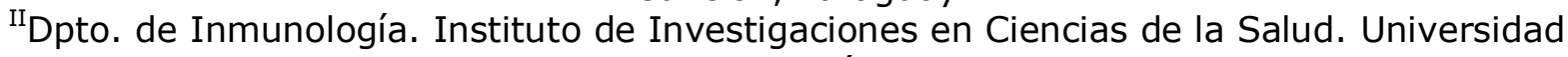 \\ Nacional de Asunción, Paraguay

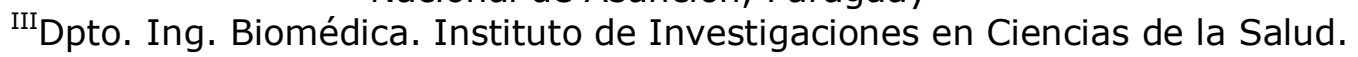 \\ Universidad Nacional de Asunción, Paraguay
}

\section{RESUMEN}

La bibliometría se refiere a la cuantificación y evaluación de la producción científica de un país, una institución, o de un autor. Son pocos los estudios bibliométricos de los investigadores paraguayos. El objetivo de este trabajo fue estudiar la producción científica de los investigadores del Instituto de Investigaciones en Ciencias de la Salud desde 1.997 al 2.011. El estudio fue de carácter retrospectivo, con un diseño observacional descriptivo de corte transversal. Se realizó una exhaustiva búsqueda de las publicaciones de los investigadores del IICS, tomando como punto de partida un artículo publicado en la revista "Memorias del IICS" en el 2.011, que lista los artículos publicados por los investigadores del IICS, que fue verificado y ampliado en las bases de datos de SciELO Paraguay, PubMeb, Lilacs y la Biblioteca Virtual en Salud (BIREME). En el periodo estudiado, fueron identificados 276 artículos científicos publicados por investigadores del IICS con una mediana de 5 autores en las revistas nacionales y 8 en las internacionales. El año con mayor producción fue el 2.009 con $30(10,9 \%)$ artículos publicados, y el año 2.000 con el menor número $(n=4)$. Con respecto a los tipos de artículos, 225 fueron originales y 180 en el idioma español. Entre las revistas nacionales la más utilizada fue "Memorias del IICS", y entre las internacionales se destaca publicaciones en revistas con elevado factor de impacto como PLoS Neglected Tropical Diseases. Los indicadores muestran que la producción del IICS sigue una línea de tendencia creciente en los últimos años.

Palabras clave: artículos indexados, bibliometría, indicadores bibliométricos, IICS-UNA, Paraguay.

\footnotetext{
ABSTRACT

Bibliometrics refers to the quantification and evaluation of the scientific production of a country, an institution or an author. There are few bibliometric studies about Paraguayan researchers. The objective of this work was to study the scientific production of the researchers of the Instituto de Investigaciones en Ciencias de la Salud (IICS in Spanish) from 1997 to 2011. This was a cross-sectional descriptive observational retrospective study. An exhaustive search of the publications of IICS' researchers was performed starting in an article published in the journal "Memorias del IICS" in 2011 that list those *Autor Correspondiente: Lic. Miguel Torales Cabañas, Perceptoria. Instituto de Investigaciones en Ciencias de la Salud. Universidad Nacional de Asunción, Paraguay

Email: miguel_asu@hotmail.com

Fecha de recepción: agosto 2014; Fecha de aceptación: enero 2015
} 
researchers. This was verified and extended in the database of SciELO Paraguay, Pub Med, Lilacs and the Health Virtual Library (BIREME). In the studied period, 276 scientific articles published by IICS' researchers with a median of 5 and 8 authors in national and international journals respectively. The most productive year was 2009 with $30(10,87 \%)$ publications, and the lowest was $2000(n=4)$. Concerning articles types, 225 were original articles and 180 were published in Spanish. Among journals, "Memorias del IICS" was the most frequent at national level while PLoS Neglected Tropical Diseases was among international ones with a high impact factor. The indicators showed that the production of the IICS has followed a growing trend in the last years.

Keywords: indexed articles, bibliometrics, bibliometric indicators, IICS-UNA, Paraguay.

\section{INTRODUCCIÓN}

El uso de indicadores bibliométricos para estudiar la actividad investigadora se basa en que las publicaciones científicas son resultado esencial de dicha actividad, y proporcionan información sobre los resultados del proceso investigador, su volumen, evolución, visibilidad y estructura. Además proporcionan herramientas útiles y objetivas en los procesos de evaluación de los resultados de la actividad científica $(1,2)$. El término "bibliometría" aparece por primera vez en el año 1.969, reemplazando la expresión "bibliografía estadística", entendida como el estudio de una disciplina científica por medio de la cuantificación y el análisis de comunicación que se difunde de la misma de forma escrita (1-5). El comportamiento de la información científica es competencia de la bibliometría. Se basa en la premisa de que las publicaciones científicas son un resultado esencial de dicha actividad (6-7). La bibliometría juega un papel crucial, pues le da un valor medible al resultado de dicha actividad científica; por consiguiente, se puede situar o comparar la creación de ' $X$ ' institución, grupo investigativo, país, en relación con otros (8).

En este sentido, la base de datos bibliográfica más utilizada a nivel mundial para la construcción de indicadores bibliométricos es el Science Citation Index (SCI), editado por el Institute for Scientific Information (ISI) de Estados Unidos, y su versión ibero e interamericana, la Red de Indicadores de Ciencia y Tecnología (RICYT), accesible actualmente a través de Internet, por medio de la plataforma virtual ISI Thompson. El SCI permite acceder a información bibliográfica, resúmenes (o abstracts) y citas de referencias de las más importantes publicaciones mundiales de ciencia y tecnología en más de 150 disciplinas como por ejemplo, microbiología, parasitología, inmunología, genética, biología molecular, patología, virología y producción de reactivos de diagnóstico in vitro.

El Instituto de Investigaciones en Ciencias de la Salud, dependiente de la Universidad Nacional de Asunción, en sus 34 años de existencia ha recibido importantes cooperaciones internacionales que permitieron el crecimiento y desarrollo institucional, la formación de un núcleo de investigadores, permitiendo la producción científica constante de la Institución y aporte de soluciones a muchas situaciones problemáticas en el país. El objetivo de este estudio fue analizar la productividad bibliométrica de los investigadores del Instituto de Investigaciones en Ciencias de la Salud entre 1.997 y 2.011.

\section{MATERIALES Y MÉTODOS}

Se realizó un estudio descriptivo transversal mediante el análisis bibliométrico de todos los artículos publicados por los investigadores del Instituto de Investigaciones en Ciencias de la Salud - IICS en revistas nacionales e internacionales. El muestreo fue de carácter censal de todos los artículos publicados en el periodo 1.997-2.011 y en los que la filiación de los docentes investigadores fuera el IICS.

No fueron incluidos los artículos publicados por los investigadores del IICS que tenían como filiación otra institución en la publicación, es decir al docente que no había 
publicado con el nombre de la institución, dejando en el anonimato los nombres de los investigadores que han publicado o no en los últimos 15 años. Se realizó una exhaustiva búsqueda de las publicaciones de los investigadores del IICS, tomando como punto de partida un artículo publicado por Kasamatsu et al. en la revista "Memorias del IICS" en el año 2.011 (5), que lista los artículos publicados en revistas nacionales e internacionales. Esta lista fue verificada en las bases de datos de SciELO Paraguay, PubMeb, Lilacs y la Biblioteca virtual en Salud (BIREME), encontrándose otros artículos publicados por los investigadores del IICS. Se utilizaron varias estrategias de búsquedas, utilizando las palabras claves Instituto de Investigaciones en Ciencias de la Salud, IICS, Paraguay, como también los nombres o apellidos de los docentes investigadores pertenecientes al IICS. En total se encontraron 276 artículos publicados, 168 en revistas nacionales y 108 en internacionales. La limitación que tuvo este procedimiento en la búsqueda de los artículos fue la falta de normalización de los nombres de los autores en las publicaciones científicas y también en los casos en que el investigador no fuese primer autor en el artículo, en donde se procedió a verificar los artículos ingresando en las revistas en las que fueron publicados y con acceso libre. El número de docentes investigadores cuya filiación corresponde al IICS encontrado en toda recuperación de documentos fue 124.

Las publicaciones fueron clasificadas por año, por procedencia de las revistas en nacionales e internacionales, y por tipo: original, reportes de casos, temas de actualidad, entre otros, así como por idiomas de publicación. Además, se clasificaron los tipos de revistas en las que fueron publicados los artículos en nacionales e internacionales.

Se analizaron las publicaciones científicas de los investigadores del IICS, teniendo en cuenta el número total de investigadores aparecidos en todas las publicaciones de las revistas, luego se clasificaron a los investigadores del IICS según su aparición como primer autor en las revistas nacionales e internacionales y por último el número total de instituciones que colaboraron en los artículos científicos del periodo estudiado. Los datos fueron analizados en una planilla electrónica de Microsoft Excel y por Epi info 2000 (CDC, Atlanta).

\section{RESULTADOS}

En el periodo de estudio (1.997-2.011) fueron identificadas 276 publicaciones científicas de los investigadores del IICS, de las cuales 168 (61\%) correspondían a artículos publicados en revistas nacionales y 108 (39\%) en revistas internacionales. Se puede observar que el mayor número fue en el año 2009 con 30 publicaciones (10,9\%). Los números más bajos de las publicaciones científicas se observan en los años $2.000(n=4)$, y $1.999(n=7)$. A excepción de estos dos años las cifras de las publicaciones se mantuvieron relativamente estables, por encima de 10 publicaciones por año, siendo la mediana de 21, y el promedio de 18 publicaciones. Esto se observa en la Tabla 1. 
Tabla 1. Distribución por año de las publicaciones científicas del IICS. Periodo 1.997-2.011. $\mathrm{n}=276$

\begin{tabular}{lccr}
\hline $\begin{array}{l}\text { Año de } \\
\text { Publicación }\end{array}$ & $\begin{array}{c}\text { Revistas } \\
\text { nacionales }\end{array}$ & $\begin{array}{c}\text { Revistas } \\
\text { internacionales }\end{array}$ & Total \\
\hline 1997 & 25 & 3 & 28 \\
1998 & 14 & 7 & 21 \\
$\mathbf{1 9 9 9}$ & $\mathbf{1}$ & $\mathbf{6}$ & $\mathbf{7}$ \\
$\mathbf{2 0 0 0}$ & $\mathbf{1}$ & $\mathbf{3}$ & $\mathbf{4}$ \\
2001 & 3 & 8 & 11 \\
2002 & 16 & 5 & 21 \\
2003 & 6 & 7 & 13 \\
2004 & 2 & 9 & 11 \\
2005 & 21 & 6 & 27 \\
2006 & 11 & 4 & 15 \\
2007 & 9 & 13 & 22 \\
2008 & 14 & 6 & 20 \\
$\mathbf{2 0 0 9}$ & $\mathbf{2 1}$ & $\mathbf{9}$ & $\mathbf{3 0}$ \\
2010 & 13 & 11 & 24 \\
2011 & 11 & 11 & 22 \\
\hline Total & $\mathbf{1 6 8}$ & $\mathbf{1 0 8}$ & $\mathbf{2 7 6}$
\end{tabular}

Las características de los artículos publicados por los investigadores tanto en revistas nacionales como internacionales se muestran en la Tabla 2, entre las cuales se destacan los artículos originales con un $81,5 \%$, seguido en forma muy distante de reporte de casos con $8,33 \%$ de las publicaciones. En relación al idioma de publicación, el español es el más prevalente observándose con una proporción del $65,2 \%$, seguido del inglés en un 34,1\%.

Tabla 2. Distribución por año y origen de la revista de las publicaciones Científicas del IICS. Periodo 1.997-2.011. $n=276$

\begin{tabular}{lrrr}
\hline Características del Artículo & $\begin{array}{c}\text { Revistas } \\
\text { Nacionales }\end{array}$ & $\begin{array}{c}\text { Revistas } \\
\text { Internacionales }\end{array}$ & Total \\
\hline Tipos de artículos & & & 108 \\
\hline Original & 117 & - & 225 \\
Reporte de casos & 23 & - & 23 \\
Temas de actualidad & 8 & - & 8 \\
Comunicación corta & 9 & - & 9 \\
Artículo de revisión & 6 & - & 6 \\
Caso clínico & 3 & - & 3 \\
Articulo especial & 1 & & 1 \\
Especificaciones Técnicas & 1 & 12 & 1 \\
\hline Idioma & & 94 & 180 \\
\hline Español & 168 & 2 & 94 \\
Inglés & - & & 2 \\
Alemán & - & & \\
\hline
\end{tabular}

En los resultados obtenidos en relación a las distintas revistas nacionales en las que fueron publicados los artículos se menciona a la revista "Memorias del IICS" con el $63,1 \%(106 / 168)$ de las publicaciones siendo la de mayor frecuencia, seguida de la revista "Annual Reports" cesante en 1998 con el 23,2\% (39/168) (Tabla 3). 
Tabla 3. Revistas nacionales. $n=168$

\begin{tabular}{lcc}
\hline Revistas nacionales & Cantidad & Porcentaje \\
\hline Memorias del IICS & 106 & 63,1 \\
Annual Reports & 39 & 23,2 \\
Revista Paraguaya de Pediatría & 15 & 8,9 \\
Anales de la Facultad de & & \\
Ciencias Médicas & 8 & 4,8 \\
\hline Annual Reports nombre anterior de Memorias del IICS
\end{tabular}

Annual Reports nombre anterior de Memorias del IICS

Se observó una gran variabilidad de las revistas internacionales utilizadas por los docentes investigadores para la publicación de sus artículos científicos. Se destacan por su mayor frecuencia "Memorias do Instituto Oswaldo Cruz" con siete $(6,5 \%)$ publicaciones seguida de "PLoS Neglected Tropical Diseases" con seis (5,6\%). Además se mencionan las revistas "The American Journal of Tropical Medicine and Hygiene" con cinco (4,6\%) y "Journal of Ethnopharmacology" $y$ "Journal of Medical Virology" con cuatro (3,7\%). Con relación al país de origen de las revistas, EE.UU fue el más frecuente con el $28,7 \%$ del total (Tabla 4 ).

Tabla 4. País de origen de las revistas internacionales. $n=108$

\begin{tabular}{lcc}
\hline País de origen & Cantidad & Porcentaje \\
\hline EEUU & 31 & 28,7 \\
Inglaterra & 16 & 14,8 \\
Brasil & 15 & 13,9 \\
Países Bajos & 10 & 9,3 \\
Alemania & 8 & 7,4 \\
*Otros & 28 & 25,9 \\
\hline *Otros: Argentina, Irlanda, Austria, Francia, México, Suiza, Chile, Japón, Bolivia, \\
Canadá, Italia y Eslovaquia.
\end{tabular}

En los datos analizados que se encuentran en la Tabla 5, se visualiza que en las publicaciones de los artículos científicos en las revistas nacionales, la mediana del número de autores fue 5 con un rango de 1 a 15 siendo ésta menor con respecto a las internacionales, cuya mediana fue de 8 y su rango de 1 a 63 autores en las publicaciones científicas.

Tabla 5. Número total de autores según la procedencia de las revistas

\begin{tabular}{|c|c|c|c|}
\hline \multirow{2}{*}{ Procedencia de la revista } & \multicolumn{3}{|c|}{ No de autores en los artículos } \\
\hline & Total & Mediana & Rango \\
\hline Nacional & 168 & 5 & $1-15$ \\
\hline Internacional & 108 & 8 & $1-63$ \\
\hline
\end{tabular}

En cuanto a la participación de varias instituciones, la Tabla 6 muestra que en las revistas nacionales la participación de una sola institución fue de 37,5\% y de dos (39,3\%) con una mediana de dos, mientras que en la internacionales en el 34,3\% fueron tres instituciones participantes con una mediana de 3 y hasta un máximo de 55 instituciones. 
Tabla 6. Número de instituciones participantes en las publicaciones según procedencia de la revista

\begin{tabular}{|c|c|c|c|c|}
\hline \multirow[b]{2}{*}{ No de Instituciones } & \multicolumn{2}{|c|}{ Nacionales } & \multicolumn{2}{|c|}{ Internacionales } \\
\hline & $\mathbf{n}$ & $\%$ & $\mathbf{n}$ & $\%$ \\
\hline 1 & 63 & $(37,5)$ & 14 & (13) \\
\hline 2 & 66 & $(39,3)$ & 19 & $(17,6)$ \\
\hline 3 & 27 & $(16,1)$ & 37 & $(34,3)$ \\
\hline 4 & 9 & $(5,4)$ & 19 & $(17,6)$ \\
\hline$\geq 5$ & 3 & $(1,8)$ & 19 & $(17,6)$ \\
\hline Mediana & & $(1-6)$ & 3 & Rango $(1-55)$ \\
\hline Total & \multicolumn{2}{|c|}{168} & & 108 \\
\hline
\end{tabular}

En las 276 publicaciones, fueron identificados 124 investigadores del IICS, de los cuales $144(85,7 \%)$ eran primer autor en artículos publicados en revistas nacionales y 51 $(47,2 \%)$ en revistas internacionales (Tabla 7$)$.

Tabla 7. Distribución de los investigadores del IICS como primer autor según la procedencia de las revistas

\begin{tabular}{lccr}
\hline Primer Autor & Nacionales & Internacionales & Total \\
\hline $\mathrm{Si}$ & $144(85,7 \%)$ & $51(47,2 \%)$ & 195 \\
No & $24(14,3 \%)$ & $57(52,8 \%)$ & 81 \\
Total & $\mathbf{1 6 8}$ & $\mathbf{1 0 8}$ & $\mathbf{2 7 6}$ \\
\hline
\end{tabular}

\section{DISCUSIÓN}

Los indicadores bibliométricos de producción son índices cuantitativos útiles para medir, entre otros, los niveles de producción científica de un país, una institución, o un autor, y en ellos influyen muchas y muy variadas circunstancias (10). Estas medidas sirven para comparar las producciones científicas entre grupos similares, pero no para evaluar la calidad de la producción de un país, una institución o una publicación (11-12). Hecha esta aclaración, se podría considerar que la producción del Instituto de Investigaciones en Ciencias de la Salud, IICS, con sus 276 trabajos en 15 años, es relativamente baja en comparación a otras instituciones de América del Sur, comparando con una institución con carácter similar al IICS, el Instituto Nacional de Salud del Perú, cuya producción científica es de 175 en tan solo siete años 2.005-2.011 (15). Si esta institución mantuviera una tendencia estable podría producir hasta 375 artículos científicos en 15 años, periodo estudiado en este trabajo. Sin embargo, se debe tener en cuenta que demográficamente el Paraguay es el segundo con menor número de habitantes de la región por encima de Uruguay, pudiendo ser éste un factor influyente al momento de comparar las producciones científicas de una institución con otra a nivel internacional.

Con respecto a lo mencionado más arriba, nuestro país era considerado de ciencia marginal hasta el 2.011 en donde se identificaron un total de 1.112 científicos, de los cuales tan solo $336(30 \%)$ tienen producción científica. Si comparamos con el periodo estudiado en este trabajo, 37\% (124/335) son investigadores del IICS, es decir un porcentaje aceptable en comparación al nivel nacional (13).

En Latinoamérica, el número de científicos por millón de habitantes es superior en: Argentina (1.091), Brasil (704), Uruguay (520) y México (384). En Paraguay, si consideramos los científicos productivos, el número sería 50. Si incluimos a quienes se consideran a sí mismos científicos (aunque no publiquen), sería 160. Para alcanzar las cifras de Uruguay y Argentina, necesitaríamos mil o dos mil nuevos científicos, respectivamente $(13)$. 
La presencia anual de las publicaciones es constante, ya que no ha habido ningún año en el que no se publicara ningún artículo científico por parte de los investigadores del IICS, descrito por estudios precedentes en los años, 2.006 (9); 2.007 (4); y 2.011 (5). Se evidencia el crecimiento gradual en los últimos años en la productividad científica de acuerdo a los indicadores de distribución por año, mostrándose prometedor el número de publicaciones futuras.

En dos ocasiones varía la tendencia de producción científica, siendo bajas en los años 2.000 , y 1.999, esto podría deberse al factor de transición que hubo a partir del año mencionado con respecto a la revista nacional perteneciente a la institución. Sin embargo, el pico más alto en todo el periodo estudiado se encontró en el 2.009, manteniéndose relativamente estable en los otros años no mencionados.

Más difícil resulta determinar si la proporción de $81,5 \%$ de artículos originales se sitúa entre los valores habituales de producción de las revistas, pues la mayoría de los artículos sobre indicadores de producción se suelen referir al conjunto de trabajos sin diferenciar por tipo de documento.

La productividad idiomática en español, prevalece como lengua dominante ante el inglés, estando la frecuencia más alta en las revistas nacionales.

En otra línea de análisis, las revistas nacionales más utilizadas por los docentes del IICS, son: "Memorias del IICS" y Annual Reports, ambas pertenecientes a la institución en la que los mismos trabajan, que muestra la endogamia en la producción científica. Con respecto a las revistas internacionales, PLoS Neglected Tropical Diseases, con un factor de impacto muy elevado de 4,57, aparece como una de las revistas de mayor frecuencia en donde los docentes investigadores del IICS publican sus artículos científicos, seguidas de otras tales como: The American Journal of Tropical Medicine and Hygiene $(2,48)$ y Memorias do Instituto Oswaldo Cruz $(1,36)$.

Los indicadores de colaboración autoral en esta investigación son muy favorables, teniendo en cuenta la participación de varios autores en una producción en cuanto indica un nivel al menos inicial de cooperación científica, sirviendo de enlace para el establecimiento de nuevas relaciones asociativas, lo que parece indicar la diversidad de conocimientos que tienen los investigadores. (14)

Cabe mencionar que en las revistas nacionales, el número de mayor frecuencia de instituciones que aparecen en los artículos publicados por los investigadores del IICS han sido dos. En tanto que en las internacionales la mayor proporción estuvo centrada en tres instituciones de los autores intervinientes, llegando incluso a 55 en un estudio multicéntrico.

Ahora bien, vale aclarar que los investigadores que publicaron con autoría del IICS, según su aparición en los artículos como primer autor son relativamente semejantes en las revistas internacionales, mientras que en las nacionales hay una marcada diferencia en la distribución, siendo el porcentaje más alto los investigadores del IICS que aparecen como primer autor.

De acuerdo con lo expresado previamente, el estudio que se ha presentado ofrece aportes significativos en el análisis de la actualidad de la producción científica de los investigadores del IICS, y una esfera más amplia en la producción como primer autor, tanto en revistas nacionales como internacionales. Aunque es necesario reconocer la limitación que tuvo el trabajo en la búsqueda de los artículos publicados por los docentes investigadores del IICS, en donde muchos de ellos eran nombrados de manera diferente en las publicaciones en las cuales tuvieron participación. Así mismo, no se ha considerado la cantidad de artículos publicados por cada uno de los investigadores en el periodo estudiado ni la antigüedad de los mismos dentro de la institución.

Aún con esto, los resultados reseñados en este artículo subrayan que desde el punto de vista cronológico las producciones científicas de los investigadores del IICS se han mantenido relativamente constantes. En cuanto a las revistas en que publican sus artículos, se destaca "Memorias del IICS", una revista nacional perteneciente a la 
institución indexada desde el año 2.005, con la totalidad de sus artículos publicadas en español debido a que los autores son en su mayoría nacionales, sin dejar de mencionar a las revistas internacionales en las que publican sus artículos y en las que se destacan revistas con factores de impacto muy elevados. Ampliando un poco más esta visión, se destaca la colaboración de varias instituciones en las publicaciones realizadas por los docentes investigadores del IICS, siendo ésta una oportunidad de trabajar en equipo, lo cual a su vez incide en la posibilidad de generar nuevas comunidades de conocimientos en las que se creen procesos de investigación.

Líneas futuras de investigación centradas en el enfoque aplicado en este estudio deberán abordar el índice de productividad de los docentes investigadores del IICS, con el fin de buscar incentivar a los docentes a seguir produciendo investigación de alta calidad, y de esta manera seguir aportando a dicha producción a nivel país para un mejor estatus a nivel mundial, ya que el Instituto de Investigaciones en Ciencias de la Salud es una de las Instituciones más prestigiosas y reconocidas a nivel país y mundial, contando con un plantel excelente de investigadores.

\section{AGRADECIMIENTOS}

Se agradece a la Dra. Graciela Velázquez, Directora del Instituto de Investigaciones en Ciencias de la Salud IICS, por el apoyo dado para la realización de este trabajo.

\section{REFERENCIAS BIBLIOGRÁFICAS}

1. Diego Camps, MD. Limitaciones de los indicadores bibliométricos en la evaluación de la actividad científica biomédica. Colomb Méd. 2008; 39(1): 74-9.

2. Camps D, Recuero $\mathrm{Y}$, Ávila RE, Samar ME. Estudio bibliométrico de un volumen de la revista Archivos de Medicina. Archivos de Medicina [Internet]. 2006 [citado 12 mayo 2012]; 2(3). Disponible en: http://archivosdemedicina.com/articlefullview/Estudio-Bibliom-trico-de-un-Volumende-la-Revista-Archivos-d_9.html

3. Rousseau R. Indicadores bibliométricos y econométricos en la evaluación de instituciones científicas. ACIMED. 2001; 9(supl.4):50-60.

4. Duarte Masi S. Indicadores bibliométricos del Paraguay. Mem. Inst. Investig. Cienc. Salud. 2006; 2(1):49-53.

5. Kasamatsu E, Casanova W, Torales F. Publicaciones científicas de investigadores del Instituto de Investigaciones en Ciencias de la Salud (IICS) de la Universidad Nacional de Asunción periodo junio 1999 - junio 2011. Mem. Inst. Investig. Cienc. Salud. 2011; 7(1): 64-82.

6. López AA, Nuñez C, Vicente-Herreros MT, Monroy N, Sarasibar H, Tejedo E. Análisis bibliométrico de la productividad científica de los artículos originales relacionados con la salud laboral publicados por diferentes revistas españolas entre los años 1997 y 2006. Medicina Balear. 2008; 23(1): 17-24. 7. Gauthier E. Bibliometric analysis of scientific and technological research: A user's guide to the methodology. Canada: Science and Technology Redesign Project, Statistics Canada; 1998.

8. Tricco AC, Runnels V, Sampson M, Bouchard L. Shifts in the use of population health, health promotion, and public health: A bibliometric analysis. Can J Public Health /Internet/. 2008 /citado 12 mayo 2012/; 99(6): 466-71. Disponible en:

http://journal.cpha.ca/index.php/cjph/article/view/1692/1876.

9. Galeano ME, Amarilla A, Parra G. Productividad científica del Paraguay en el área de biomedicina: un análisis bibliométrico. Mem. Inst. Investig. Cienc. Salud. 2007; 5(1):26-30.

10. Bordons M, Zulueta MA. Evaluación de la actividad científica a través de indicadores bibliométricos. Rev Esp Cardiol. 1999; 52(10):790-800.

11. Llana Martín A, Barredo Sobrino MP. Estudio bibliométrico de la producción científica de los departamentos básicos de la Facultad de Medicina de la Universidad Autónoma de Madrid: 19902000 /Internet/. Madrid: Universidad Autónoma de Madrid /citado 12 mayo 2012/. Disponible en: https://repositorio.uam.es/bitstream/handle/10486/234/22388_cc10.pdf?sequence=1.

12. Ardanuy J. Breve introducción a la bibliometría /Internet/. Barcelona: Universitat de Barcelona; 2012 /citado 12 mayo 2012/. Disponible en:

http://diposit.ub.edu/dspace/bitstream/2445/30962/1/breve\%20introduccion\%20bibliometria.pdf. 
13. Cubilla AL. Pocos científicos y baja productividad. /Sitio Web/. Asunción: Portal Guaraní; 2011 [actualizado 2013; acceso en febrero 2014]. Disponible en:

http://www.portalguarani.com/2677_antonio_I_cubilla/21984_pocos_cientificos_y_baja_productivid ad_por_dr_antonio_I_cubilla.html

14. Ávila-Toscano JH, Marenco-Escuderos A, Madariaga Orozco C. Indicadores bibliométricos, redes de coautorías y colaboración institucional en revistas colombianas de psicología. Avances en Psicología Latinoamericana. 2014; 32(1):167-82.

15. Redalycs. Red de Revistas Científicas de América Latina y el Caribe, España y Portugal. Indicadores cienciométrico: Una mirada alternativa a la medición científica de instituciones y países [Sitio Web]. México: Universidad Autónoma del Estado de México; 2001. [actualizado 2014; acceso 17 mayo 2014]. Disponible en: http://www.redalyc.org/IndicadoresHome.oa. 